\title{
Throughput Analysis of Nonbinary Type-II Hybrid ARQ
}

\author{
Lijun Zhang \\ Dept. of Electronic Engineering \\ Tsinghua University \\ Beijing, 100084, CHINA \\ Email: zhanglijun99@tsinghua.org.cn
}

\author{
Victor O. K. Li \\ Dept. of Electrical and \\ Electronic Engineering \\ The University of Hong Kong \\ Hong Kong, CHINA \\ Email:vli@eee.hku.hk
}

\author{
Zhigang Cao \\ Dept. of Electronic Engineering \\ Tsinghua University \\ Beijing, 100084, CHINA \\ Email: czg-dee@tsinghua.edu.cn
}

\begin{abstract}
Nonbinary type-II hybrid ARQ (HARQ), which combines shortened Reed-Solomon (RS) code with ARQ, is proposed. Its throughput is obtained by extending Lin and Yu's analysis of binary type-II HARQ. Analytical results show that nonbinary HARQ outperforms its binary counterpart in throughput over Rayleigh fading channels when the modulation scheme and the FEC subsystem are selected properly.
\end{abstract}

\section{INTRODUCTION}

In wireless communications, automatic-repeat-request (ARQ) and forward-error-correction (FEC) are the two basic schemes for error control. ARQ can provide very high reliability, but the throughput cannot be guaranteed under adverse channel conditions. On the contrary, FEC maintains a constant throughput (which equals the coding rate), but the reliability is unsatisfactory in bad channel states. It seems natural to combine them, and the hybrid ARQ (HARQ) [1] came into being. Much work has been done in the field [2]-[7]

In an HARQ system, the FEC subsystem reduces the frequency of retransmission by correcting the error patterns which occur most frequently, thus increasing the system throughput. If an error pattern exceeds the error-correction capability of FEC, the receiver requests a retransmission instead of passing the unreliably decoded message to the user. HARQ can provide higher throughput than ARQ alone, and almost the same reliability of ARQ if proper codes are chosen.

In this paper, Nonbinary type-II HARQ, which combines shortened Reed-Solomon (RS) code with ARQ, is proposed. Being a maximum-distance-separable (MDS) code, RS code has very flexible coding rate adjustment and powerful error correction capacity. In addition, this nonbinary block code can be easily mapped to the nonbinary modulation constellation, increasing the bandwidth utilization of the system.

\section{DESCRIPTION OF LIN AND YU'S BINARY TYPE-II HARQ}

In type-II HARQ, two linear codes are used [8]. One is a high rate $\left(n_{0}, k_{0}\right)$ code $C_{0}$, which is designed for error detection only. The other is a half-rate invertible $\left(2 k_{1}, k_{1}\right)$ code $C_{1}$, which is used for error-correction and error-detection simultaneously. In the binary case, $k_{0}=k_{1}$. The procedure of type-II HARQ is as follows.

When a message $D$ is ready for transmission, it is encoded into a codeword $(D, Q)$ based on the error-detecting code $C_{0}$, where $Q$ represents the parity-check part. At the same time, $D$ is also encoded by code $C_{1}$ into $(D, P(D))$, where $P(D)$ is the redundant part. At first, codeword $(D, Q)$ is transmitted and the received word is $(\tilde{D}, \tilde{Q})$. If no errors are detected, the word will be assumed error-free and accepted by the receiver; otherwise, the erroneous message $\tilde{D}$ is saved and a negative acknowledgment (NACK) is sent back to the source. Upon receiving this NACK, the transmitter encodes the parity-check part $P(D)$ into a codeword $\left(P(D), Q^{(1)}\right)$ based on $C_{0}$, and sends the parity word. The received parity word $\left(\tilde{P}(D), \tilde{Q}^{(1)}\right)$ will be also examined according to $C_{0}$. If no errors are detected, the message $D$ is retrieved by the invertible process. If errors are detected, we combine $\tilde{P}(D)$ with the previously saved $\tilde{D}$ to correct errors. If the error pattern in $(\tilde{D}, \tilde{P}(D))$ is correctable, the message $D$ is recovered and accepted by the receiver; otherwise, $\tilde{D}$ is discarded and $\tilde{P}(D)$ is buffered. At the same time, the second NACK is sent back for information word retransmission. The retransmission alternates between the parity and the information words until the message is received error-free, or recovered by inversion, or by the decoding process.

\section{EXTENSION TO NONBINARY HARQ}

Shortened RS codes replace binary codes as $C_{1}$ in nonbinary HARQ. Now, we prove the validity of the invertible process for RS $\left(2 k_{1}, k 1\right)$.

RS codes are MDS codes, and have the property $d_{\text {min }}=$ $n-k+1$, where $n, k$, and $d_{\min }$ are the code length, information length, and minimum distance of the codes, respectively. When a linear code is shortened, its distance property remains unchanged [1]; thus $d_{\text {min }}=k_{1}+1$. If all the paritycheck symbols are error-free, we assume that the information symbols are all zeros. The assumption causes at most $k_{1}$ symbol errors. As opposed to normal errors, these man-made errors' locations are known. Such errors can be corrected as long as the number of errors is less than $d_{\text {min }}$. Obviously, $k_{1}<d_{m i n}$, and the invertible process works. 


\section{THROUGHPUT}

Assume that the size of both modulation constellation and $C_{0}$ 's alphabet is $M$, and RS $\left(2 k_{1}, k_{1}\right)$ is defined over $\mathrm{GF}\left(2^{l m}\right)$, where $m=\log _{2} M$. Each symbol of this RS code consists of $l m$ bits. When mapping the code symbol to channel symbol, each code symbol is represented by the concatenation of $l$ consecutive channel symbols. Using this mapping, long and powerful RS codes can be combined'with small-constellation modulations [9]. Obviously

$$
k_{0}=l k_{1} \text {. }
$$

Let $p_{0}$ and $p_{1}$ represent channel symbol error rate and code $C_{1}$ 's symbol error rate, respectively. Assuming the channel symbol errors occur independently, we have $p_{1}=1-\left(1-p_{0}\right)^{l}$. Let $Q_{1}$ be the conditional probability that a message $D$ is recovered successfully from the first received parity block $\tilde{P}(D)$ either by the inversion process or by the decoding process on the code $C_{1}$, given that errors are detected in the received word $(\tilde{D}, \tilde{Q})$. Through some basic probability transform, we derive

$$
Q_{1}=p_{c}+\frac{\Phi_{1}+\Phi_{2}+\Phi_{3}+\Phi_{4}}{1-p_{c}-p_{e}}
$$

where $p_{c}$ and $p_{e}$ are the probabilities that a $C_{0}$-coded word will be transmitted error-free and include undetectable error patterns, respectively. Obviously,

$$
p_{c}=\left(1-p_{0}\right)^{n_{11}}
$$

The undetectable error probability $p_{e}$ can be ignored if $C_{0}$ is properly selected. If we set error correction capability of the RS code to $t, \Phi_{1}$ through $\Phi_{4}$ are

$$
\begin{aligned}
& \Phi_{1}=\operatorname{Pr}(\text { Case I) } \\
& \Phi_{2}=\operatorname{Pr}(\text { Case II) } \\
& \Phi_{3}=\operatorname{Pr}(\text { Case III), } \\
& \Phi_{4}=\operatorname{Pr}(\text { Case IV). }
\end{aligned}
$$

The definitions of Case I through IV are as follows.

Case I At least one error in $\tilde{D}$, at least one error in $\tilde{P}(D)$, and the total number of errors in $(\tilde{D}, \tilde{P}(D))$ do not exceed $t$.

Case II No errors in $\tilde{D}$, at least one error in $\tilde{Q}$, and the number of errors in $\tilde{P}(D)$ are at least one, but no more than $t$.

Case III No errors in $\tilde{P}(D)$, at least one error in $\tilde{Q}^{(1)}$, and the number of errors in $\tilde{D}$ are at least one, but no more than $t$.

Case IV No errors in $(\tilde{D}, \tilde{P}(D))$, at least one error in $\tilde{Q}$, and at least one error in $\tilde{Q}^{(1)}$.

All the cases are shown in Fig. 1. For simplicity of expression, we define

$$
\Delta_{i} \triangleq\left(\begin{array}{c}
k_{1} \\
i
\end{array}\right) p_{1}^{i}\left(1-p_{\perp}\right)^{k_{1}-i} \quad i=0,1, \cdots, k_{1} .
$$

'We choose the same notations as in Lin and $\mathrm{Yu}$ [8].

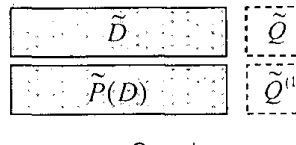

Case I

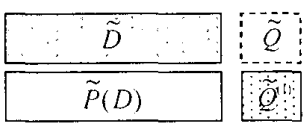

Case. III

Error free
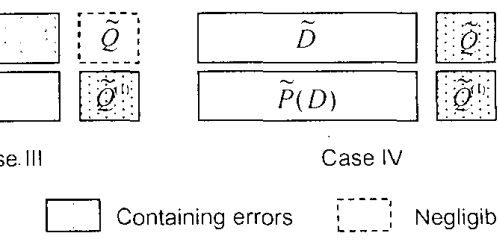

Case IV

Fig. 1. Error distribution of all the cases.

According to the error distribution shown in Fig. 1, we get

$$
\begin{aligned}
& \Phi_{1}=\sum_{i=2}^{t} \sum_{j=1}^{i-1} \Delta_{j} \Delta_{i-j} \\
& \Phi_{2}=\Phi_{3}=\left(1-p_{1}\right)^{k_{1}}\left[1-\left(1-p_{0}\right)^{n_{(1}-k_{1}}\right] \sum_{i=1}^{t} \Delta_{i} \\
& \Phi_{4}=\left(1-p_{1}\right)^{2 k_{1}}\left[1-\left(1-p_{0}\right)^{n_{(1)}-k_{k_{1}}}\right]^{2} .
\end{aligned}
$$

When we ignore $p_{e}$ and let $X=\Phi_{1}+\Phi_{2}+\Phi_{3}+\Phi_{4}, Q_{1}$ becomes

$$
Q_{1}=p_{c}+\frac{X}{1-p_{c}} .
$$

Let $Q_{2}$ be the conditional probability that a message $D$ is successfully recovered from the second received information word $\left(\tilde{D^{\prime}}, \tilde{Q}^{\prime}\right)$ given that errors are detected in the received parity word $\left(\tilde{P}(D), \tilde{Q}^{(1)}\right)$ and the first received information word $(\tilde{D}, \tilde{Q})$, and that the received parity word $\left(\tilde{P}(D), \tilde{Q}^{(1)}\right)$ fails to recover $D$, but detects the presence of errors in $(\tilde{D}, \tilde{P}(D)) . Q_{2}$ can be expressed as

$$
Q_{2}=p_{c}+\frac{1}{\left(1-p_{c}\right)\left(1-Q_{1}\right)} \sum_{i=1}^{t-1} \sum_{j=t-i+1}^{k_{1}} \sum_{l=1}^{t-i} \Delta_{i} \Delta_{j} \Delta_{l}
$$

Let $Y=\sum_{i=1}^{t-1} \sum_{j=t-i+1}^{k_{1}} \sum_{l=1}^{t-i} \Delta_{i} \Delta_{j} \Delta_{l}$, we can rewrite $Q_{2}$ as

$$
Q_{2}=p_{c}+\frac{Y}{\left(1-p_{c}\right)\left(1-Q_{1}\right)} .
$$

The buffer size $N$ of the receiver is set to the number of $C_{0}$-coded words transmitted during the roundtrip delay $\tau$ at information rate $c$, namely

$$
N=\frac{c \tau}{m k_{0}} .
$$

Resorting an inferior system [8], we get the lower bound of the throughput. Due to space limitations, only the final results are presented. The details of the derivation are similar to that in [8]. The throughput $\eta$ of the nonbinary type-II HARQ with selective-repeat (SR) is lower bounded as follows:

$$
\eta \geqslant m \cdot \frac{k_{0}}{n_{0}} \cdot \frac{\delta_{0}}{\delta_{0}+\delta_{1}+N \delta_{2}} .
$$


where

$$
\begin{aligned}
\dot{\delta}_{0} & =\frac{B_{3}}{1-B_{2}}\left(1-B_{3} B_{2}^{N-1}\right), \\
\dot{\delta}_{1} & =p_{c} Q_{1} B_{1}^{N-2}+p_{c}\left(1-Q_{1}\right) Q_{2} B_{2}^{N-2}, \\
\dot{\delta}_{2} & =2-p_{c} Q_{1} B_{1}^{N-2}-B_{1} B_{3} B_{2}^{N-2},
\end{aligned}
$$

and

$$
\begin{aligned}
& B_{1}=p_{c}\left(2-p_{c}\right)+X \\
& B_{2}=1-\left(1-p_{c}\right)^{3}+\left(1-p_{c}\right) X+Y, \\
& B_{3}=B_{1}+\frac{Y}{1-p_{c}} .
\end{aligned}
$$

We observe that the results of the nonbinary case resemble that of Lin and Yu's binary case. If $p_{1}=p_{0}$ and $m=1,(11)$ reduces to the binary case, which is identical with (35) in [8].

$$
\text { V. ANALYTICAL RESULTS }
$$

Two nonbinary HARQs are considered in the calculation, with QPSK and 8PSK modulations, respectively. Information is firstly encoded by shortened RS $(62,31)$ code to get information part and parity check part separately. Then 12 parity bits are appended to these two parts individually, which are generated by the following cyclic generator polynomial:

$$
g_{\mathrm{crc12}}=D^{12}+D^{11}+D^{3}+D^{2}+D+1 .
$$

The roundtrip delay $\tau$ and data rate $c$ are assumed to be $33 \mathrm{~ms}$ [10] and $2 \mathrm{Mbps}$, respectively. Rayleigh fading channel with perfect symbol interleaving is assumed and the channel symbol error rate (identical with the symbol error rate of $C_{0}$, i.e. $p_{0}$ ) can be expressed approximately

$$
p_{0} \approx 1-\sqrt{\frac{\sin ^{2}\left(\frac{\pi}{M}\right) \gamma}{1+\sin ^{2}\left(\frac{\pi}{M}\right) \gamma}},
$$

where $\gamma$ denotes the average signal to noise ration (SNR) of the received signal.

Figs. 2 illustrates the throughput of nonbinary type-II HARQ with QPSK modulation. For comparison, the throughput of the ideal SR ARQ with infinite receiver buffer is also presented. In the whole SNR range, the HARQ outperforms the SR ARQ in throughput, and the throughput also increases with the error-correction capacity $t$, which can be observed clearly from the figure. The nonbinary HARQ with 8PSK modulation has a similar trend and is not mentioned again.

Binary and nonbinary HARQs are compared in Fig. 3, where the binary HARQ using shortened BCH $(1000,500)$ code is just what proposed by Lin and $\mathrm{Yu}$ in [8]. Obviously, the nonbinary HARQ schemes are superior to the binary case when they have the same error-correction capability. When $t=$ 10, the nonbinary HARQs with QPSK and 8PSK modulation intercross each other around SNR of $19 \mathrm{~dB}$. Before the point, QPSK modulation can provide higher throughput than 8PSK modulation, and 8PSK modulation will exceed after the point. For $t=15$, the similar phenomenon occurs near the SNR of $16 \mathrm{~dB}$. This shows that the nonbinary HARQs with different modulations can compensate each other, thus helping extract the maximal throughput potential.

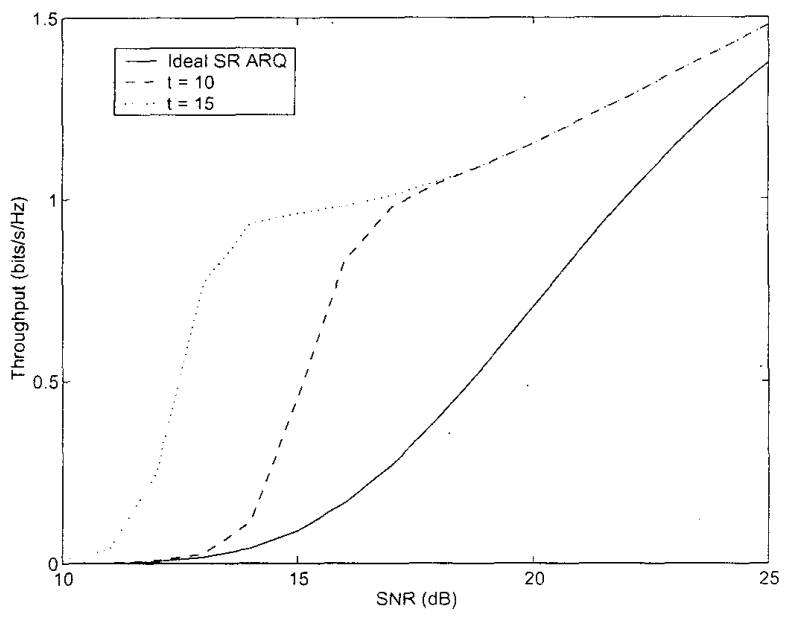

Fig. 2. Throughput comparison between ideal SR ARQ and nonbinary type-II HARQ with QPSK modulation in Rayleigh fading channels.

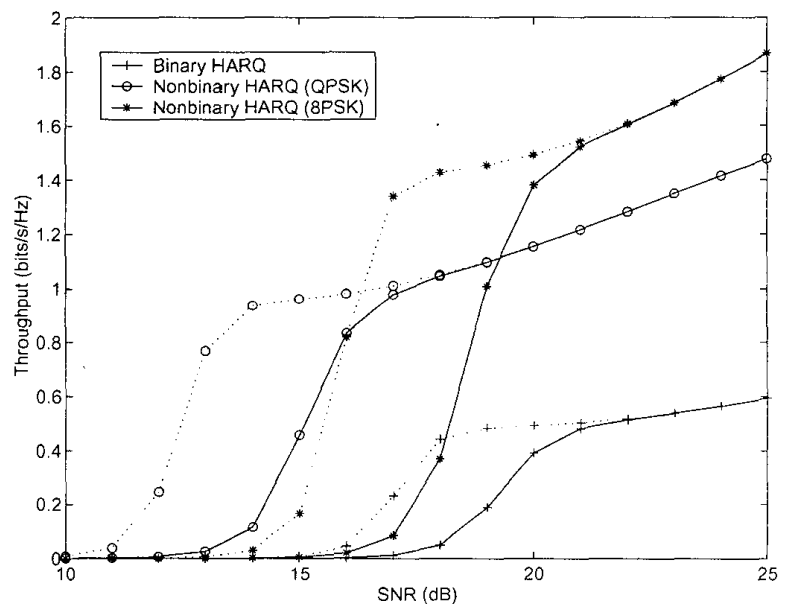

Fig. 3. Throughput comparison between binary and nonbinary HARQs in Rayleigh fading channels: solid lines for $t=10$ and dotted lines for $t=15$

\section{CONCLUSIONS}

Nonbinary type-II HARQ, which combines shortened RS code with ARQ, is proposed. Throughput of the HARQ with finite buffer is analyzed. Two nonbinary HARQs with QPSK and 8PSK, respectively, are examined in Rayleigh fading channels. The numerical results show that nonbinary HARQ is superior to the ideal SR ARQ, and also outperform its binary counterpart by a large margin.

\section{ACKNOWLEDGMENT}

This research is supported in part by the National Natural Science Foundation of China (No.6001161943)/Research Grants Council of Hong Kong Joint Research Scheme under Grant No. N_HKU 709/00. 
The $14^{\text {th }}$ IEEE 2003 International Symposium on Personal, Indoor and Mobile Radio Communication Proceedings

\section{REFERENCES}

11] S. Lin, and D. J. Costello Jr. Error Control Coding: Fundamentals and Applications, Englewood Cliffs, N.J: Prentice-Hall, 1983.

|2] K. H. Deng, and M. L. Lin, "A type I hybrid ARQ system with adaptive code rates," IEEE 7irans. Commun., vol. COM-43, pp. 733737. Feb./Mar/Apr. 1995.

[3] S. Falahati, and A. Svensson, "Hybrid Type-Il schemes with adaptive modulation systems for wireless channels," Proc. IEEE VTC'99 Fall, vol. 5. pp. 2691-2695, 1999.

[4] S. Kallel. "Efticient hybrid ARQ protocols with adaptive forward error correction," IEEE Trans. Commun., vol. COM-42, pp. 281-289, Feb.Mar.Apr. 1994

[5] Y. Chang. "A new adaptive hybrid ARQ scheme," IEEE Trans. Commum., vol. COM-43, pp. 2169-2171, Jul. 1995.
[6] M. A. Kousa, and M. Rahman, "An adaptive error control system using hybrid ARQ schemes," IEEE Trans. Commun., vol. COM-39, pp. 1049 1057, Jul. 1991.

[7] A. Shiozaki, "Adaptive type-II broadcast ARQ system." IEEE Truns. Commun., vol. COM-44, pp. 420 422. Apr. 1996

8] S. Lin, and P. S. Yu, "A hybrid ARQ scheme with parity retransmission for crror control of satellite channels." IEEE Trans. Commun., vol COM-30, pp. 1701-1719, Jul. 1982

[9] S. H. Jamali, and T. Le-Ngoc, Coded-Modulation Techniques for Fading Channels, Kluwer Academic Publishers, 1994.

[10] S. Aramvith, C. W. Lin. S. Roy, and M. T. Sun. "Wireless video transport using conditional retransmission and low-delay interleaving," IEEE Trans. Circuit. and System. for Video Tech., vol. 12, pp. 558-565, Jun. 2002. 